\title{
Two Valid Measures of Self-rated Physical Activity and Capacity
}

\author{
Marit Sundal Holen ${ }^{\mathrm{a}}$, Rønnaug Een ${ }^{\mathrm{a}}$, Thomas Mildestvedt ${ }^{\mathrm{a}}$, Geir Egil Eide ${ }^{\mathrm{a}, \mathrm{b}}$ and Eivind Meland ${ }^{\mathrm{a},{ }^{*}}$ \\ ${ }^{a}$ Department of Public Health and Primary Health Care, Research Group for General Practice, University of Bergen, \\ Bergen, Norway \\ ${ }^{b}$ Center for Clinical Research, Haukeland University Hospital, Bergen, Norway, and Department of Public Health and \\ Primary Health Care, Lifestyle Epidemiology Research Group, University of Bergen, Bergen, Norway
}

\begin{abstract}
Objectives: Questionnaires on physical activity (PA) and physical capacity (PC) are valuable tools, as they are cost beneficial, and have high response rates. The validity of short versions of such questionnaires has not been examined satisfactorily. Therefore, we aimed at examining the validity of a set of questions coding for PA and PC.

Design: The questions were administered to 217 men and women attending a cardiac rehabilitation program. Participants also gave blood samples, measuring HDL cholesterol, triglycerides (TG), insulin, glucose, and microCRP. The relations between PA and PC and biological markers were examined by linear regression analyses.

Results: Measures for PC and for PA were identified by factor analysis, which proved internally consistent. TG, homeostatic model assessment (HOMA) score, and mCRP were all significantly associated with the measures of PC and PA.

Conclusions: The measures of PA and PC are valid compared with biological markers, allowing cost-beneficial and timeefficient evaluation of important measures for cardiovascular health.
\end{abstract}

Keywords: Cardiac rehabilitation, motor activities, outcome assessment, physical fitness, questionnaires.

\section{INTRODUCTION}

Physical activity (PA) and physical capacity (PC) are inversely associated with the risk of cardiovascular disease [1], colorectal cancer, diabetes, stroke [2,3], and mortality [4]. PA and PC, the latter also named cardio respiratory fitness, are interrelated, but the impact on coronary and cardiovascular disease differs. While disease risk diminishes linearly with increasing PA, there is a precipitous decrease in risk when comparing the lowest to the next lowest category of PC [1].

Several methods to determine the degree of PA and PC exist, including observer-dependent methods such as double labeled water, calorimeters, heart rate monitoring, ventilation, cardio respiratory fitness, body temperature, motion sensors, and behavioral observation, and self-report methods such as records, recalls, logs, psychophysical rating scales, and questionnaires [5]. Compared to the other methods, questionnaires are easy to administer and distribute and, if short and focused, allow the collection of data from a large number of study subjects, with a high response rate.

Various types of questionnaires for PA and PC exist, and these have been more or less validated. Kurtze et al. [6]

*Address correspondence to this author at the Department of Public Health and Primary Health Care, University of Bergen, Kalfarveien 31, N-5018 Bergen, Norway; Tel: +4755586155; Fax: +4755586130;

E-mail: Eivind.Meland@isf.uib.no suggested that self-rated PA in Norwegian population studies, lacked satisfactory validation. Others have pointed out that the lack of practical, valid, reliable, and sensitive instruments for PA assessment have limited research in an important area [5].

The objective of this study was accordingly to validate a limited set of questions concerning PA and PC with exercise sensitive biochemical markers.

\section{MATERIAL AND METHODS}

In a two-year period from September 2000 to August 2002, a group of 266 patients attended a cardiac rehabilitation program at Krokeide Rehabilitation Center in Bergen, Norway. The program lasted for four weeks, and the patients, almost exclusively suffering from coronary heart disease (205 of 217), were voluntarily recruited to the rehabilitation stay either from hospital or from their general practitioners (GPs). The study was performed as a randomized controlled trial with two groups, and in this observational study we combine the two cohorts, and do not compare the two groups. Details on recruitment, clinical treatment, measurements, inclusion and exclusion criteria, and drop-outs are given in a former paper [7].

\section{Participants}

A total of 217 patients agreed to participate and were included in the trial. Written informed consent was obtained. 
During the two years of follow up some chose to leave the rehabilitation program or were lost to follow-up. Table $\mathbf{1}$ gives background characteristics and the measures used for validation of the self-rated PA and PC scores. The table also shows valid responses at the start of the rehabilitation stay (T0) and conclusion of the study (T24).

At $\mathrm{T} 0$ and $\mathrm{T} 24$, the patients completed a questionnaire covering multiple topics, including age, gender, and smoking status, and questions on PA, PC, and emotional status. At T24 they received and returned the questionnaire by mail.

Fasting blood samples at T0 and T24 were obtained and mailed to the study group for preparation and preservation in an ultra-freezer at minus $80^{\circ} \mathrm{C}$. Insulin, glucose, triglycerides, high density lipoprotein (HDL) cholesterol and micro C-reactive protein (mCRP) were all measured with approved methodology at a research laboratory at Haukeland University Hospital, and with low and acceptable analytic coeffi- cients of variation $[8,9]$. All analyses were performed in one run to reduce analytic variance.

To measure insulin resistance, we used the original homeostatic model assessment (HOMA1) model from Matthews et al. [10]. Emotional problems in the aftermath of a myocardial infarction have been related to subsequent morbidity and mortality [11]. Therefore, state-dependent feelings of anxiety, depression, and irritability were assessed by a validated and reliability tested Anxiety-DepressionIrritability (ADI) questionnaire [11]. The ADI score comprises 12 pairs of adjectives rated on a seven-level Likert scale. Smoking status was assessed by a single item with two response options (yes or no).

Table 2 shows the questions relating to PC (questions 14) and those relating to PA (questions 5-9). The response options and ratings are also shown in the table. Questions 7-9 have previously been used in epidemiological surveys in

Table 1. Characteristics of the 217 Patients from the Rehabilitation Program at Krokeide Rehabilitation Center, Bergen, Norway (2000 to 2002)

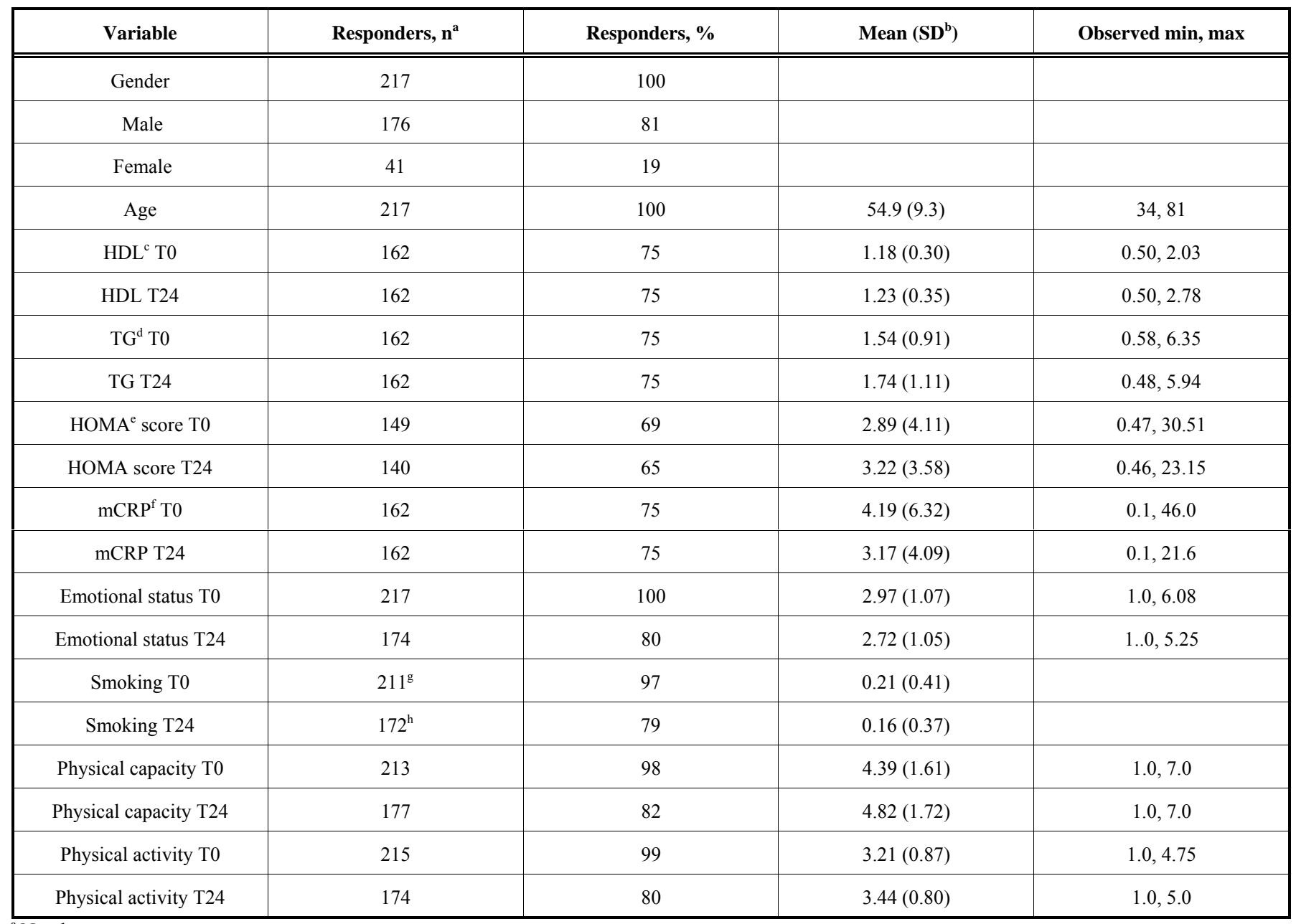

${ }^{a}$ Number

${ }^{\mathrm{b}}$ Standard Deviation

${ }^{\mathrm{c}}$ High density lipoprotein

${ }^{\mathrm{d}}$ Triglycerides

${ }^{\mathrm{e}}$ The HOMA score is explained in the text under Material and Method

${ }^{\mathrm{f}}$ Micro c-reactive protein

${ }^{\mathrm{g}} 44$ smokers among 211 responders $(21 \%)$

h 28 smokers among 172 responders $(16 \%)$ 
Table 2. Questions Coding for Physical Capacity and Physical Activity, with Response Options

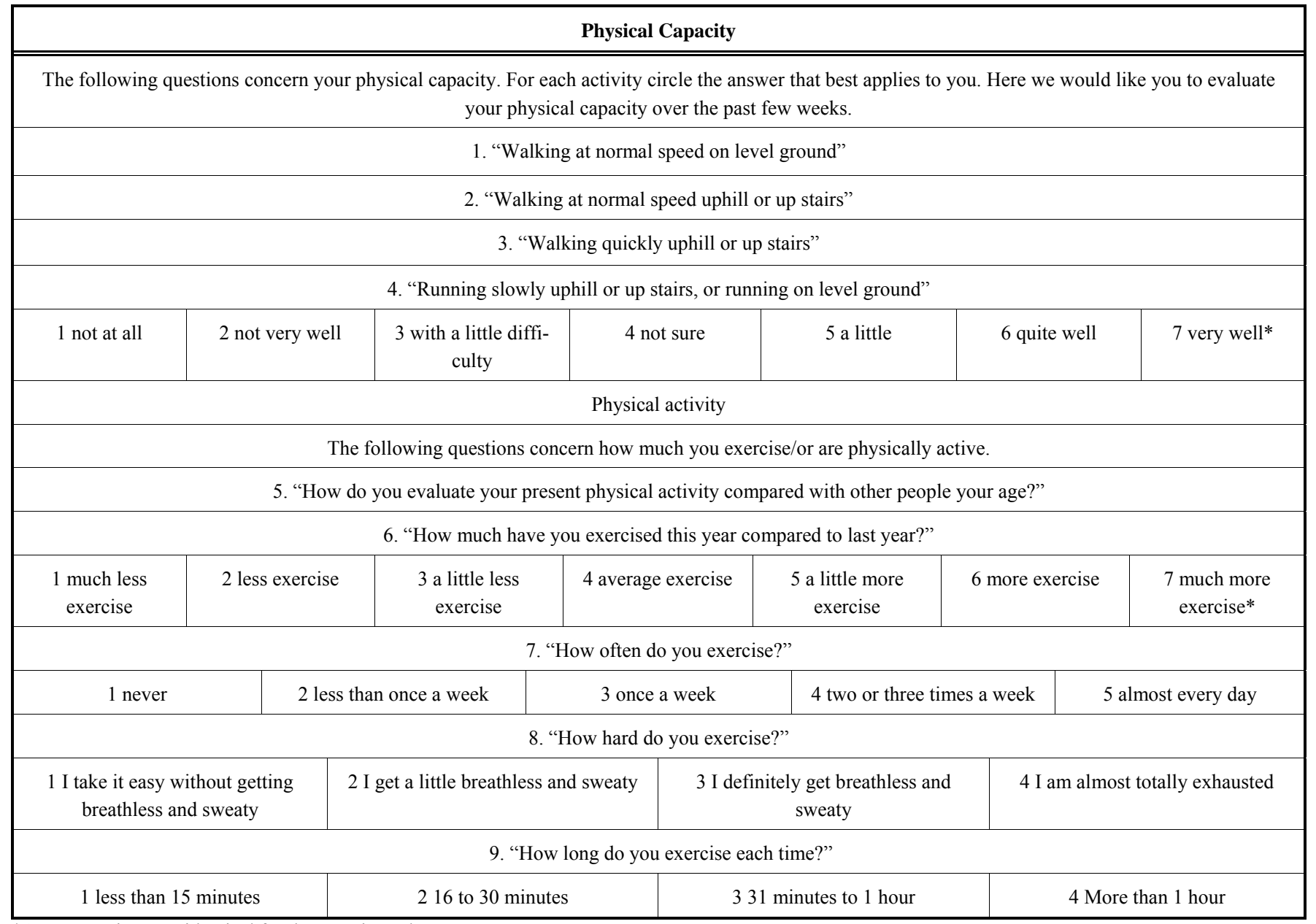

*Answer options are identical for the questions above

Norway (The Nord Trøndelag Health Surveys, HUNT) [12]. The questions have also been tested for reliability and validity [12].

Questions 5 and 6 are taken from the Stanford Five City Project, and have been tested with satisfactory concurrent validity [13]. Question 5 also proved valid in a primary care setting with male patients with a high risk of coronary heart disease [14]. The physical capacity score, also known as Maximal Physical Ability, has been validated previously in a Norwegian post-infarction study [15].

We validated the questions on PA and PC by comparing these measures with biochemical markers. These biochemical markers were chosen as they are all correlated with coronary heart disease [16-18], and are also affected by exercise. Glucose homeostasis is quite consistently associated with levels of exercise $[19,20]$. Exercise has also shown an improvement in the lipid profile, especially the triglycerides (TG) and the HDL cholesterol levels [19, 21, 22]. PA also reduces the levels of mCRP, but the literature is not fully consistent $[23,24]$.

\section{Ethics}

The study was approved by the Regional Committee for Medical Research Ethics, Health Region III, and the Norwegian Data Inspectorate.

\section{Statistical Analyses}

To evaluate how the questions relate to each other, we performed a factor analysis using Oblimin rotation. This rotation was used as this method allows conceptual and statistical association between the measures that are tested. We computed the mean scores of PC (questions 1-4) and PA (questions 5, 7-9). Reliability analysis was performed for PC and PA, respectively, and the Cronbach's alphas are reported for the composite mean scores.

We used linear regression analysis to evaluate the associations of PA and PC with the biological measures. The analyses were computed both unadjusted and adjusted, at T0 and T24. Adjustments were made by controlling for age, gender, smoking status, and ADI, all plausible confounders in this context. We also performed linear regression analyses, evaluating the sensitivity for changes of the self-rated measures, comparing them with changes of biochemical markers over the two years. Changes of PA were finally compared with changes of PC using Pearson's correlation coefficient.

From the analyses of residuals from the regression models it was found that a deviation from normality was significant in 23 out of 32 cases, mostly due to negative skewness. However, with the high number of observations $(n=107$ to 
162) asymptotic normality of the estimated regression coefficient was fairly achieved. The assumption of variance homogeneity was not seriously violated in any case. Statistical power was satisfactory, but was estimated based on the twogroup comparison in the randomized trial [25]. SPSS version 15.0 was used for the analyses.

\section{RESULTS}

Table 1 presents background characteristics and the measures used for validation of the self-rated PA and PC scores. Two hundred seventeen participants (176 men) with a mean age of 55 years volunteered. Response rates varied between 65 and 99 percent for the different measures, as shown in Table 1.

\section{Factor and Reliability Analyses}

From the factor analysis two factors were identified with acceptable convergent and discriminating ability. Questions 1-4 (Table 2), constituting the component for PC, showed high loadings with values $>0.79$. The discriminating validity was satisfying with the loadings for factor 2 having absolute values $<0.3$ for all four questions. Questions 5, 7, 8, and 9 (Table 2) had loadings $>0.67$ for the PA component, and thus showed adequate loadings for this factor. The discriminating validity was also satisfying with all values for component 1 being $<0.3$. Question 6 had identical and low loadings for both components and was therefore excluded from further validation analyses. We computed the mean scores for PC from questions 1-4, and those for PA from questions 5, 7, 8, and 9. The Cronbach's alphas for PC and PA were 0.92 and 0.72 , respectively. Both measures thus had satisfactory internal consistency.

\section{Regression Analyses, Associations with the Biochemical Markers}

Table 3 shows that both PA and PC, adjusted and unad- justed, were significantly associated with mCRP, with unstandardized regression coefficients (B) ranging from to -1.67 to -1.52 at T0. Except for unadjusted PA, there were also significant associations with the HOMA score, with B values ranging from -0.93 to -0.63 . For TG we found significant associations except for adjusted PA, with B varying from -0.22 to -0.10 . HDL cholesterol, on the other hand, was not significantly associated with PA or PC, neither adjusted nor unadjusted.

At T24 (Table 4) we found significant associations between $\mathrm{PA}$ and $\mathrm{PC}$ and $\mathrm{mCRP}$, with $\mathrm{B}$ values ranging from -1.67 to -0.73 , and $\mathrm{TG}$, with $\mathrm{B}$ values ranging from -0.53 to -0.10 . For HDL cholesterol the $\mathrm{B}$ value related to $\mathrm{PA}$ ranged from 0.08 to 0.10 in the unadjusted and the adjusted analyses respectively, and for the HOMA score the $\mathrm{B}$ values ranged from -1.12 to -0.33 . Both HDL cholesterol and the HOMA score were significantly related with PA, adjusted and unadjusted, but not with PC.

Tables 3-4 also present the explained variance $\left(\mathrm{R}^{2}\right)$ for the measures included in the regression models. Although the self-rated measures are significantly associated with the biological measures, they explain the variations in biological markers rather modestly ( $\mathrm{R}^{2}$ of 0.15 or lower).

We also performed regression analyses for the associations between differences in self-ratings and changes in biological measures over the two years. No significant associations were found. Lastly, we performed a correlation analysis of two year PA change with PC change, yielding a Pearson's correlation coefficient of $0.35(p=0.01)$.

\section{DISCUSSION}

The present study found that both the internal and discriminating validity of the questions on PA and on PC are good and the composite measures show good reliability. We identified mCRP, TG, and the HOMA score as the measures most consistently related with PC and PA. MicroCRP has

Table 3. Associations between Physical Activity and Physical Capacity and Biological Measures Analyzed with Linear Regression, Unadjusted and Adjusted ${ }^{\mathrm{i}}$ at $\mathrm{TO}$

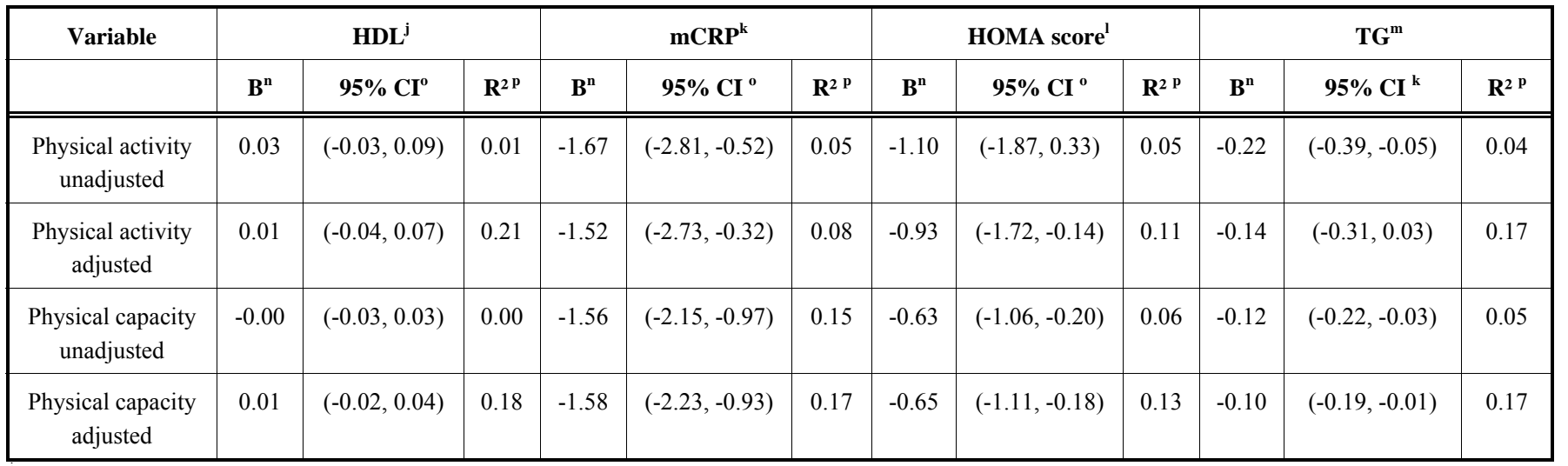

${ }^{i}$ Adjusted analyses with control for age, gender, smoking status, and emotional distress

${ }^{\mathrm{j}}$ High density lipoprotein

${ }^{\mathrm{k}}$ Micro c-reactive protein

${ }^{1}$ The HOMA score is explained in the text under Material and Method

$\mathrm{m}$ Triglycerides

${ }^{\mathrm{n}} \mathrm{B}$ : Unstandardized regression coefficient

${ }^{\circ} \mathrm{CI}$ : Confidence interval

${ }^{\mathrm{p}} \mathrm{R}^{2}$ : Determination coefficient 
Table 4. Associations between Physical Activity and Physical Capacity and Biological Measures Analyzed with Linear Regression, Unadjusted and Adjusted ${ }^{\mathrm{i}}$ at T24

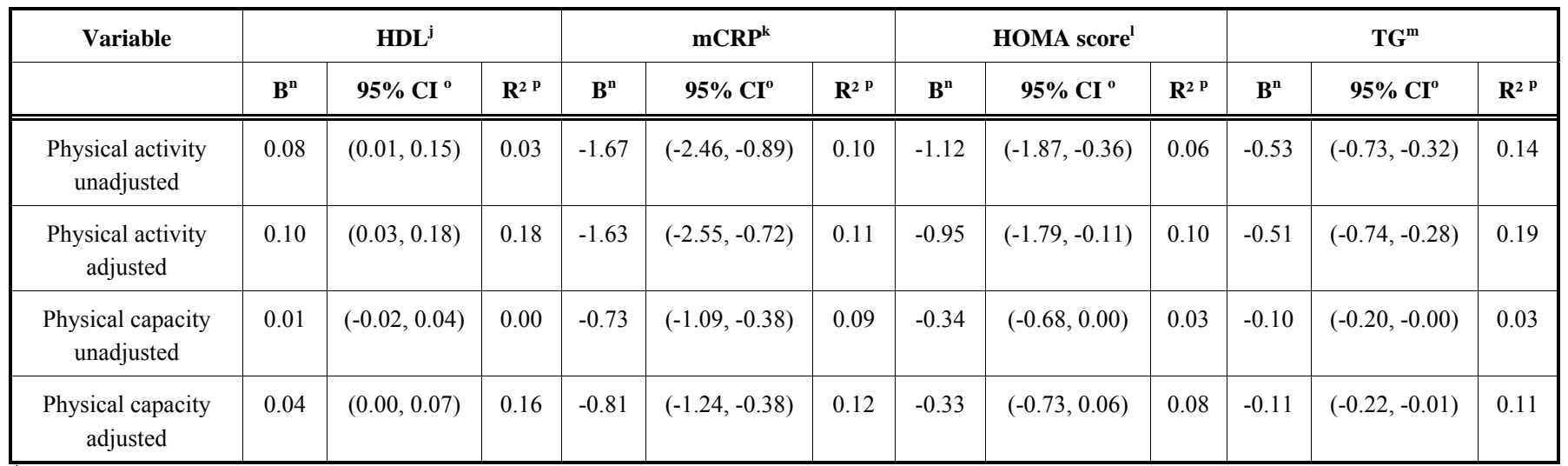

${ }^{1}$ Adjusted analyses with control for age, gender, smoking status, and emotional distress

${ }^{\mathrm{j}}$ High density lipoprotein

${ }^{\mathrm{k}}$ micro C- reactive protein

${ }^{1}$ The HOMA score is explained in the text under Material and Methods

${ }^{\mathrm{m}}$ Triglycerides

${ }^{\mathrm{n}} \mathrm{B}$ : Unstandardized regression coefficient

${ }^{\circ} \mathrm{CI}$ : Confidence interval

${ }^{\mathrm{p}} \mathrm{R}^{2}$ : Determination coefficient

significant associations with PA and PC at both observation times; TG also exhibits significant associations, except for the adjusted association of PA at T0; and the HOMA score was also significantly associated with PA and PC, though more inconsistently than the other two measures. These biochemical markers, especially mCRP, also explained the variation most strongly. Self-rated PC explained 3 to 15 percent, and self-rated PA 4 to 14 percent of the variation of $\mathrm{mCRP}$ and TG levels.

Sensitivity to intra-individual changes was not confirmed by the comparison with biochemical markers. However, the measures of biochemical markers hardly changed during the observation. The sensitivity was, though, confirmed by a moderate correlation between PA and PC change. The internal validity of the study may have suffered from the lack of control for the medications the participants were taking. However, patients with coronary heart disease are consistently treated with statins, beta blockers and acetylsalicylic acid during hospital stay in Norway, and we therefore maintain that the internal validity was safeguarded.

The diet of the participants might also affect the biochemical markers. For instance, omega-3 fatty acids lower TG and increase HDL [26]. In addition, we regrettably did not have measures of the participants' weights or BMI. A further possible source of error is that the participants, although strongly advised to, may not have met fasting with unintended elevations in blood glucose, free fatty acids, and triglycerides as a result [27].

There are three common sources of error when using biomarkers: issues relating to biological specimen collection; processing and storage; and laboratory error and withinperson variability [28]. We safeguarded factors associated with analytic variability, but the control of within-person variability was not fully satisfactory. Although reliability testing was not performed, three of the questions on PA, 7, 8, and 9 , and the PC questions have formerly been subjected to test-retest measurements, demonstrating satisfactory reliability $[12,15]$.

We are fully aware that the present study is not a complete validation study. Content validity seems well documented by high response rates and few missing answers for the questions pertaining to PA and PC. Judging the items for PA and PC by qualitative evaluation, we maintain that they seem relevant for exercise and cardiovascular health. Therefore, content validity seems safeguarded. As the PA and PC measures are associated with exercise sensitive biological markers, we also maintain that construct validity is documented. The study does not, however, address convergent validity adequately. To do so we should have compared the measures with a proven gold standard. In lack of such a gold standard, we might claim that the significant correlation between PA and PC change during the observation period seems a useful surrogate for the evaluation of convergent validity.

There are few validation studies for PA and PC with biochemical markers. In a study from the Stanford Five City Project, Blair and coworkers [13] found significant associations between changes in reported energy expenditure and changes in HDL and TG levels. Likewise, in a study on 4386 men and women, validating two questions on whether the participants performed strenuous PA on a regular basis, Haskell et al. [29] also found significant associations between HDL levels and reported levels of PA. In accordance with our findings, studies reveal that exercise reduces insulin resistance and improves glucose control in both healthy people and patients with diabetes mellitus (DM) type $2[19$, 20].

In a German cardiovascular prevention study, the authors found significant associations between the reported level of PA and HDL and TG levels [30]. Our results are consistent with these findings although the associations with HDL were 
weaker. Lack of statistical power and statin drug therapy are probable explanations for this.

In accordance with our findings, a study of patients attending a cardiac rehabilitation exercise training program, demonstrated significantly decreased median levels of $\mathrm{mCRP}$ with increasing PA [24]. Among younger and healthy persons, no effects on mCRP were observed from exercise [31], whereas another study of healthy but older participants with higher levels of mCRP, more frequent physical activity was independently associated with a lower odds of having an elevated mCRP level [23].

Questions 7, 8, and 9 from the HUNT1 study were earlier validated by Kurtze et al. [12]. The summary index of the questions was moderately correlated with $\mathrm{VO} 2 \mathrm{max}$, with motion registration, with minutes spent in vigorous $\mathrm{PA}$, and with estimated METs from the International Physical Activity Questionnaire (IPAQ) [12]. As our composite PA score is strongly impacted by the HUNT1 questions, the present study also confirms the validity among older patients with cardiac disease.

Question 5 was earlier validated in the Five City Project Community Health Survey [13]. They found, for both women and men, associations between rated activity level from the seven-day recall questionnaire and question 5 [13]. We confirmed that this question is strongly associated with other valid measures of PA.

Questions 1-4, the components in the PC measure, were earlier validated in two studies of cardiac patients [15, 25]. The measure had acceptable concurrent and construct validity, and the results of the present study confirm the validity of this measure.

A frequently used PA questionnaire is the IPAQ, which was developed by a multinational group in the late 1990s, supported by the WHO. Compared to the IPAQ short version, the questionnaire validated in this project is about the same size, short and easily manageable. It is selfadministered, and thus less resource demanding. Our questionnaire contains the same three dimensions as the IPAQ short version: intensity, time, and frequency of PA. Unlike the IPAQ, it also includes the dimension of PC, and thus is more relevant for studies focusing cardiovascular health.

In conclusion, our study demonstrates that self-rated and brief measures of PA and PC are valid compared with several exercise sensitive biological markers. We have identified four questions coding for PA and four questions coding for PC with good validity and internal consistency that correspond especially with mCRP, TG, and the HOMA score. Such questions can be used as estimates of individual PA and PC, allowing cost-beneficial and time-efficient evaluation of important measures of cardiovascular health in clinical and epidemiological research.

\section{CONFLICTS OF INTEREST}

The authors confirm that this article content has no conflicts of interest.

\section{ACKNOWLEDGEMENTS}

We would like to thank Svein Folmo, Randi Johansen, Bent Folkvord, and Arne Huus at the Krokeide Rehabilita- tion Center for their participation as clinical staff in this study. The Center is funded by the Norwegian Association of Heart and Lung Patients. This project has been financed with the aid of the Norwegian Association of Heart and Lung Patients and the EXTRA funds from the Norwegian Foundation for Health and Rehabilitation. We are also grateful to the patients at Krokeide who voluntarily participated in this study.

\section{FUNDING}

Norwegian Association of Heart and Lung Patients and EXTRA funds from the Norwegian Foundation for Health and Rehabilitation, Grant nr 2002/2/0071.

\section{REFERENCES}

[1] Williams PT. Physical fitness and activity as separate heart disease risk factors: a meta-analysis. Med Sci Sports Exerc 2001; 33(5): 754-61.

[2] Ellekjaer H, Holmen J, Ellekjaer E, Vatten L. Physical activity and stroke mortality in women. Ten-year follow-up of the NordTrondelag health survey, 1984-1986. Stroke 2000; 31(1): 14-8.

[3] Nilsen TI, Vatten LJ. Prospective study of colorectal cancer risk and physical activity, diabetes, blood glucose and BMI: exploring the hyperinsulinaemia hypothesis. Br J Cancer 2001; 84(3): 417-22.

[4] Blair SN, Kampert JB, Kohl HW, et al. Influences of cardiorespiratory fitness and other precursors on cardiovascular disease and all-cause mortality in men and women. JAMA 1996; 276(3): 205-10.

[5] Lagerros YT, Lagiou P. Assessment of physical activity and energy expenditure in epidemiological research of chronic diseases. Eur J Epidemiol 2007; 22(6): 353-62.

[6] Kurtze N GK, Holmen J. Self-reported physical activity in population studies - a methodological problem. Nor J Epidemiol 2003; 13(1): 163-70.

[7] Mildestvedt T, Meland E. Examining the "Matthew Effect" on the motivation and ability to make lifestyle changes in 217 heart rehabilitation patients. Scand J Public Health 2007; 35(2): 140-7.

[8] Lazarevic G, Antic S, Cvetkovic T, Vlahovic P, Tasic I, Stefanovic V. A physical activity programme and its effects on insulin resistance and oxidative defense in obese male patients with type 2 diabetes mellitus. Diabetes Metab 2006; 32(6): 583-90.

[9] Anderson JW, Zettwoch N, Feldmann T, Tietyen-Clark J, Oeltgen P, Bishop CW. Cholesterol-lowering effects of psyllium hydrophilic mucilloid for hypercholsterolemic men. Arch Intern Med 1988; 148: 292-6.

[10] Wallace TM, Levy JC, Matthews DR. Use and abuse of HOMA modeling. Diabetes Care 2004; 27(6): 1487-95.

[11] Havik OE, Maeland JG. Patterns of emotional reactions after a myocardial infarction. J Psychosom Res 1990; 34(3): 271-85.

[12] Nanna Kurtze VR, Bo-Egil Hustvedt, Dana Flanders. Reliability and validity of self-reported physical activity in the Nord Trøndelag Health Study - HUNT 1. Scand J Pub Health 2008; 36: 52-61.

[13] Blair SN, Haskell WL, Ho P, et al. Assessment of habitual physical activity by a seven-day recall in a community survey and controlled experiments. Am J Epidemiol 1985; 122(5): 794-804.

[14] Meland E, Laerum E, Ulvik RJ. Effectiveness of two preventive interventions for coronary heart disease in primary care. Scand $J$ Prim Health Care 1997; 15(1): 57-64.

[15] Maeland JG, Havik OE. Self-assessment of health before and after a myocardial infarction. Soc Sci Med 1988; 27(6): 597-605.

[16] Pyorala K, De Backer G, Graham I, Poole-Wilson P, Wood D. Prevention of coronary heart disease in clinical practice. Recommendations of the Task Force of the European Society of Cardiology, European Atherosclerosis Society and European Society of Hypertension. Eur Heart J 1994; 15(10): 1300-31.

[17] O'Donovan G, Kearney EM, Nevill AM, Woolf-May K, Bird SR. The effects of 24 weeks of moderate- or high-intensity exercise on insulin resistance. Eur J Appl Physiol 2005; 95(5-6): 522-8.

[18] Backes JM, Howard PA, Moriarty PM. Role of C-reactive protein in cardiovascular disease. Ann Pharmacother. 2004; 38(1): 110-8. 
[19] Thompson PD, Crouse SF, Goodpaster B, Kelley D, Moyna N, Pescatello L. The acute versus the chronic response to exercise. Med Sci Sports Exerc 2001; 33(6 Suppl): S438-45; discussion S523.

[20] Goodyear LJ, Kahn BB. Exercise, glucose transport, and insulin sensitivity. Annu Rev Med 1998; 49: 235-61.

[21] Lippi G, Schena F, Salvagno GL, Montagnana M, Ballestrieri F, Guidi GC. Comparison of the lipid profile and lipoprotein(a) between sedentary and highly trained subjects. Clin Chem Lab Med 2006; 44(3): 322-6.

[22] Kelley GA, Kelley KS, Tran ZV. Aerobic exercise and lipids and lipoproteins in women: a meta-analysis of randomized controlled trials. J Womens Health (Larchmt) 2004; 13(10): 1148-64.

[23] Abramson JL, Vaccarino V. Relationship between physical activity and inflammation among apparently healthy middle-aged and older US adults. Arch Intern Med 2002; 162(11): 1286-92.

[24] Milani RV, Lavie CJ, Mehra MR. Reduction in C-reactive protein through cardiac rehabilitation and exercise training. J Am Coll Cardiol 2004; 43(6): 1056-61.

[25] Mildestvedt T, Meland E, Eide GE. How important are individual counselling, expectancy beliefs and autonomy for the maintenance of exercise after cardiac rehabilitation? Scand J Publ Health 2008; 36: $832-40$.
[26] Balk EM, Lichtenstein AH, Chung M, Kupelnick B, Chew P, Lau J. Effects of omega-3 fatty acids on serum markers of cardiovascular disease risk: a systematic review. Atherosclerosis 2006; 189(1): 19-30.

[27] O'Keefe JH, Bell DS. Postprandial hyperglycemia/hyperlipidemia (postprandial dysmetabolism) is a cardiovascular risk factor. Am J Cardiol 2007; 100(5): 899-904.

[28] Tworoger SS, Hankinson SE. Use of biomarkers in epidemiologic studies: minimizing the influence of measurement error in the study design and analysis. Cancer Causes Control 2006; 17(7): 889-99.

[29] Haskell WL, Taylor HL, Wood PD, Schrott H, Heiss G. Strenuous physical activity, treadmill exercise test performance and plasma high-density lipoprotein cholesterol.The Lipid Research Clinics Program Prevalence Study. Circulation 1980; 62(4 Pt 2): IV53-61.

[30] Mensink GB, Heerstrass DW, Neppelenbroek SE, Schuit AJ, Bellach BM. Intensity, duration, and frequency of physical activity and coronary risk factors. Med Sci Sports Exerc 1997; 29(9): 11928.

[31] Campbell KL, Campbell PT, Ulrich CM, et al. No reduction in Creactive protein following a 12 -month randomized controlled trial of exercise in men and women. Cancer Epidemiol Biomarkers Prev 2008; 17(7): 1714-8.

(C) Holen et al.; Licensee Bentham Open.

This is an open access article licensed under the terms of the Creative Commons Attribution Non-Commercial License (http://creativecommons.org/licenses/ by-nc/3.0/) which permits unrestricted, non-commercial use, distribution and reproduction in any medium, provided the work is properly cited. 